\title{
Theoretical Inspection of TM-P4C Single-Atom Electrocatalyst: Ultra-High Performance for bifunctional Oxygen Reduction and Evolution Reactions
}

\author{
Chaohong Guan, Hong Zhu* \\ University of Michigan-Shanghai Jiao Tong University Joint Institute, Shanghai Jiao Tong \\ University, Shanghai 200240, China \\ Email: hong.zhu@sjtu.edu.cn
}

\begin{abstract}
Developing the cost-effective or even bifunctional electrocatalysts for both oxygen evolution reaction (OER) and oxygen reduction reaction (ORR) with industrially relevant activity is highly desired for metal-air batteries at the current stage. Herein, in this work, the catalytic performances of the single transition metal (TM) atom embeds graphene sheet with the tetra-coordinates Phosphorus (TMP4) for ORR and OER were investigated based on the density functional theory method. The results demonstrate that the most promising ORR and OER catalytic activity can achieved on the CoP4 with the smallest potential gap $\Delta E$ and the lowest overpotentials of 0.37 and $0.32 \mathrm{eV}$ among all TMP4 systems, respectively, and the catalytic activity is even better than that of the traditional $\mathrm{Pt}$ and $\mathrm{IrO}_{2}$ catalysts. Furthermore, the AIMD calculation was conducted to confirm the thermodynamics stability of CoP4. This work screens out promising candidates for novel graphene-based bifunctional ORR and OER catalysts and provides the theoretical guidance for the development of single-atom catalysts.
\end{abstract}

Keywords: Oxygen evolution reaction, oxygen reduction reaction, TMP4, Density functional theory

\section{1 introduction}

At present, the continuously growing environmental pollution and energy depletion greatly inspire the development of renewable energy technologies including Li-ion batteries [1], fuel cells [2] and metal-air batteries [3], etc., which have emerged as systems of commercial and notable scientific interests. Among them, the rechargeable metal-air batteries $[4,5]$ have obtained the increasing concerns due to the features in environment, cost and particularly in the potential 
application to replace the traditional Li-ion batteries which theoretical capacity is largely limited by the electrode materials (to date, the theoretical capacity has nearly reached) [6]. Typically, owing to the use of organic and aqueous electrolytes the metal-air batteries are divided into two groups, like Li-air batteries and $\mathrm{Zn}$-air batteries, respectively. The former still needs breakthroughs in the issue of reaction products block subsequent reactions [7]; the latter is regarded as the safer and more reliable system, whose electrochemical reaction rate largely relies on the electrocatalytic processes of oxygen reduction reaction (ORR) and oxygen evolution reaction (OER) at cathodes $[8,9]$. Previous studies $[10,11]$ have symbolized Pt-based catalysts are the most efficient for the ORR process, while the Ir- or Ru-based catalysts, for the OER, show promising catalytic activity. However, the mentioned catalysts before contains the noble metals so that the expensive cost is a fundamental obstacle for their large-scale practical application [12]. In this scenario, developing the cost-effective or even bifunctional electrocatalysts for both ORR and OER with industrially relevant activity is highly desired for metal-air batteries at the current stage.

Recently, two-dimensional transition metal (TM) single atom catalysts (SACs) for metal-air batteries have received tremendous attention attributed to the low cost, high specific surface area, unique chemical and physical characteristics [13], such as transition-metal disulfide dichalcogenides [14], two-dimensional (2D) metal-organic frameworks [15], etc. And intensive efforts have been made to explore/improve their ORR or OER activity and inspiring achievements have been made in recent years [16,17]. For instance, Li and colleagues [14] demonstrated the $\mathrm{Cu}$-doped MoS2 exhibits the best ORR performance by theoretical screening the TM doped-MoS2 surfaces. Deng et al. [18] comprehensive studied the catalytic activity of 2D transition metal based tetracyanoquinodimethane (TM-TCNQ) monolayers and indicated that the OER activity of Ni-TCNQ is better than Fe-TCNQ, which is regarded as the most promising candidate. Also, as the most popular carbon-based 2D materials, graphene, has shown desired bifunctional catalytic activity for both ORR and OER when a TM atom was loaded on the surface [19-22]. Specifically, TM-N-C systems derived from graphene have been designed theoretically and experimentally to be one of the most favorable catalytic materials, the single TM atom coordinated with $\mathrm{N}$ atoms is regarded as the active center and promotes the high catalytic activity [23-26]. For the theoretical researches of TM-N-C system, the first principle based on the density functional theory (DFT) is typically adopted to screen and explore the materials with high catalytic performance [27-29]. For 
example, simulations indicated CoN4-gra system has been developed to have a stronger active with the overpotential of $0.69,0.47 \mathrm{eV}$ for ORE and ORR than $\mathrm{CoN}_{\mathrm{X}-\mathrm{gra}}(\mathrm{x}=1-3)$ systems, exhibiting the comparably activity with the noble metal catalysts [30]. The FeN4-gra also shows more promising ORR activity than FeNX-gra systems [31]. Lu et al. [32] theoretically proposed the MnN4-gra system and suggested its ORR activity is prior than FeN4-gra. And the catalytic mechanisms of other TM atoms embedded graphene with $\mathrm{N}$ coordination, such as $\mathrm{Zn}$ [33], $\mathrm{Cu}$ [34], Ni [35], have also been explored in recent years. In the experimental aspect, many TM-N-C catalysts (TM including $\mathrm{Fe}, \mathrm{Ni}, \mathrm{Zn}, \mathrm{Co}$, etc.) [36-38] have been practically synthesized and also exhibit promise ORR/OER activity. As Sun et al. [39] developed a highly active Cu-N-C catalyst through the pyrolysis of $\mathrm{Cu}(\mathrm{NO} 3) 2 \cdot 3 \mathrm{H} 2 \mathrm{O}$ and urea, showing an excellent ORR activity (5.36 mA $\mathrm{cm}^{-2}$ at $0.9 \mathrm{~V} ; \mathrm{E}_{1 / 2}=0.898 \mathrm{~V}$ vs RHE). Therefore, these results indicate that the kind of structure of TM-N-C system offers a great opportunity for the practical application of ideal ORR/OER catalysts, and the design of novel catalysts with higher activity and stability need to be challenged continuously.

As shown in periodic table, phosphorus $(\mathrm{P})$ occupies the same group with $\mathrm{N}$ element and possesses the comparable properties. Further, P-doped carbon materials with the prospect of high catalytic activity are of thrive interests owing to the lower electronegativity and longer covalent radius than $\mathrm{N}$-doped carbon, which illustrates that $\mathrm{P}$ doping facilitates the structural and active modification of carbon. So far, experiment results have proved P-doped structures with different carbon, including graphene [40], mesoporous carbon [41] and graphite layers [42], etc., possess the improved ORR activity. However, few works have been conducted on the catalytic performance for P and metal atoms co-doped carbon materials. Yang et al. [43] synthesized the Co and P co-operated mesoporous carbon and demonstrated its ORR activity is superior to the carbon with Co-doping or P-doping. Subsequently, they developed the P and iron co-doped carbon but the ORR activity is lower than that of Co and P co-doped carbon [44]. The P and Fe-functionalized graphene prepared by Razmjooei and co-workers exhibits a high comparable ORR activity to commercial Pt-based catalysts [45]. In addition, theoretical studies also reveal that TM-P-C $(\mathrm{TM}=$ $\mathrm{Fe}, \mathrm{Co}, \mathrm{Ni}$ ) is a novel promising catalyst for $\mathrm{CO}$ oxidation with the determined catalytic energy barriers of $0.87,0.81$ and $0.79 \mathrm{eV}$, respectively.

To our best knowledge, few studies have been reported to comprehensively explore the 
bifunctional ORR/OER activity of transition metal and $\mathrm{P}$ co-doped graphene-based catalysts with the determined TM-P coordination numbers (TM-P4-C) to date. There is a large gray area exists in evaluating the feasibility and revealing the detailed catalytic mechanism of TM-P4-C on ORR or OER, the more appropriate TM atom for catalytic processes and the origin of the ORR/OER activities remain unclear. Herein, to breakthrough these obstacles, we introduced single TM atom (from $\mathrm{Ti}$ to $\mathrm{Au}$ ) with the coordination of $\mathrm{P}$ into the graphene sheet as the SACs, pioneeringly and comprehensively studied its bifunctional ORR and ORE activity by first-principles calculation.

\section{Computational Details}

Spin-polarized first-principle calculations were implemented by Vienna ab initio simulation package (VASP) [46] with an energy cutoff of $500 \mathrm{eV}$, the Perdew-Burke-Ernzerhof (PBE) functional within the generalized gradient approximation (GGA) was adopted to address the exchange-correlation interactions [47,48]. The $\mathrm{P}$ and TM doped $5 \times 5 \times 1$ graphenes were modeled with a vacuum thickness of $15 \AA$ and optimized with a $\Gamma$ centered $2 \times 2 \times 1$ k-point sampling, specially, the $5 \times 5 \times 1$ k-point was employed to calculate the density of states (DOS). During the optimized process, all atoms were relaxed until the convergence criterions of energy $(1 \times 10-6 \mathrm{eV})$ and force $\left(0.02 \mathrm{eV}^{-1}\right)$ are reached. DFT-D3 developed by Grimme was utilized for the van der Waals correction [49]. During the DFT calculations, the implicit solvent model was employed to consider the solvation effect [50]. In addition, AIMD simulations were carried out to explore the thermodynamic stability of TM-P4-C catalysts at 300K.

The Gibbs free energy changes $(\Delta \mathrm{G})$ of oxygen-containing reaction intermediates on TM-P4-C are defined as [51]:

$$
\Delta \mathrm{G}=\Delta \mathrm{E}+\Delta Z E P-T \Delta S+\Delta U
$$

Where $\triangle \mathrm{E}$ is the energy calculated by density functional theory (DFT) method, ZEP and $\mathrm{S}$ are the zero-point energy and entropy, respectively, which can be obtained by the frequency analysis through VASPKIT package (summarized in Table S1). T is set as the room temperature of 298.15 $\mathrm{K}$. In addition, the influence of applied electrode potential is considered by the term of $\Delta U$.

Binding energies of TM-P4-C will be confirmed by the $E_{b}$ as shown in following [52]:

$$
E_{b}=\left(E_{T M-P 4-C}-E_{P 4-C}-E_{T M}\right)
$$

in which $E_{T M-P 4-C}$ is the total energy of TM atom with the coordination of P embedded into 
grphene sheet, $E_{P 4-C}$ and $E_{T M}$ represent the energy of P4-C and TM atom, respectively. And the adsorption free energy of reaction intermediates on TM-P4-C surfaces was determined by [51]

$$
\begin{gathered}
\Delta E_{\text {ads }}=E_{\mathrm{O}^{*} / \mathrm{OH}^{*} / \mathrm{OOH}^{*}}-E_{*}-E_{\mathrm{O} / \mathrm{OH} / \mathrm{OOH}} \\
E_{\mathrm{O}}=E_{\mathrm{H}_{2} \mathrm{O}}-E_{\mathrm{H}_{2}} \\
E_{\mathrm{OH}}=E_{\mathrm{H}_{2} \mathrm{O}}-1 / 2 E_{\mathrm{H}_{2}} \\
E_{\mathrm{OOH}}=2 E_{\mathrm{H}_{2} \mathrm{O}}-3 / 2 E_{\mathrm{H}_{2}}
\end{gathered}
$$

where $E_{O^{*} / O H^{*} / O O H^{*}}$ and $E_{*}$ represent the total energy of TM-P4-C with and without the adsorption of oxygen-containing groups, $E_{\mathrm{O} / \mathrm{OH} / \mathrm{OOH}}$ can be calculated according to the energies of $\mathrm{H}_{2} \mathrm{O}$ and $\mathrm{H}_{2}$ molecules.

\section{Results and discussion}

\subsection{Related intermediates adsorption on TM-P4-C catalysts}

Recently, TM and P co-doped carbon SACs have caught extensive attentions due to the similar properties with $\mathrm{N}$ and exhibit the resembled configurations with TM-N4-C systems, as displayed in Figure 1a, the single TM atom embeds graphene sheet with the tetra-coordinates $\mathrm{P}$ (TMP4). Via DFT calculations, for the final optimized structures (the corresponding stable configurations are illustrated in Figure S1), the details of binding energies $\left(\mathrm{E}_{\mathrm{b}}\right)$, and TM-P bond lengths are listed in Table $S 2$, the negative values of $E_{b}$ certify the stability of TMP4. Meanwhile, the bader charge analysis was conducted to evaluate the strong interactions between P4-C and TM atoms (Table S2). And the transferred charges from TM atoms to P4-C induce to the TM atom charged positively, hence, the site of charged TM will be regarded as the adsorption and catalytic centers during the ORR/OER process. Figure $1 \mathrm{~b}$ depicts the DOS of P4-C and TiP4, more electronic states will be introduced to the gap near the Fermi level by the d orbitals of TM atoms when they embed to P4-C, which promotes the increased electric conductivity and may devoted to strengthening the ORR/OER electrocatalytic performance. 

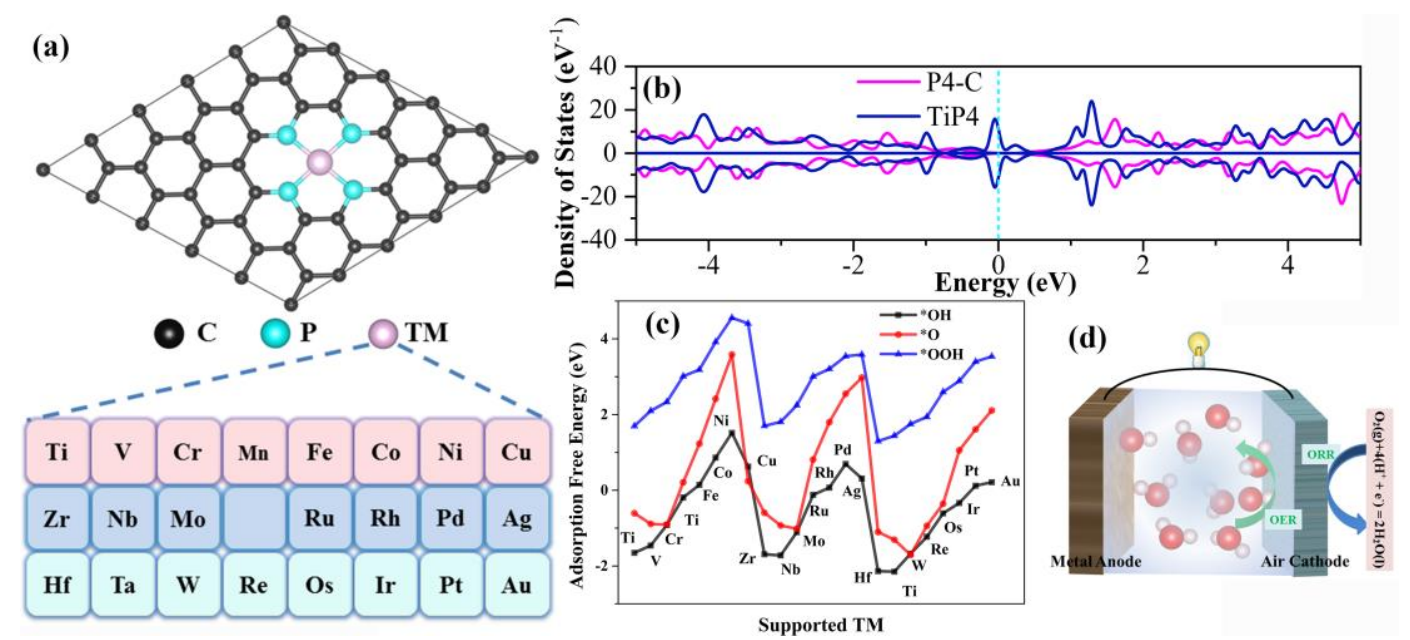

Figure 1. (a) Top view of P4-C with a transition metal atom (from Ti to $\mathrm{Au}$ ) doped in the center, black, blue and pink represent the carbon, phosphorus and transition metal atom, respectively. (b) Density of states (DOS) of P4-C and TiP4 with the Fermi level referred to $0 \mathrm{eV}$. (c) Comparison of adsorption free energies of related intermediates (OH, O, OOH) on TMP4. (d) Schematic model of a metal-air battery.

For the ORR/OER process, the adsorption free energies of $\mathrm{OH}^{*}\left(\Delta E_{O H^{*}}\right), \mathrm{O}^{*}\left(\Delta E_{O^{*}}\right)$ and $\mathrm{OOH}^{*}\left(\Delta E_{O O H^{*}}\right)$ on catalyst surface determine the free energy of each reaction step. As a matter of common knowledge, the ideal catalyst will provide a $1.23 \mathrm{~V}$ reaction free energy for every step of ORR/OER with no overpotential, thus, for designing the novel catalysts, the suitable adsorption free energies of intermediates should be guaranteed to maintain the low reaction overpotential. In this work, the adsorption free energies for $\mathrm{OH}^{*}, \mathrm{O}^{*}$ and $\mathrm{OOH}^{*}$ on TMP4 are compared and listed in Figure 1c, which clearly demonstrates that the $\Delta E_{a d s}$ of species containing oxygen are determined by the optimized TMP4 structures. Moreover, it can be confirmed that the TMP4 catalysts prefer to capturing oxygen with the relatively lower $\Delta E_{O H^{*}}$ ranging from -2.15 to $1.5 \mathrm{eV}$. For all intermediates adsorption on TMP4, the changes of free energy are similar with the increased TM atomic numbers ( $3 \mathrm{~d}, 4 \mathrm{~d}$ and $5 \mathrm{~d}$ elements). The strongest chemical bond formed between $\mathrm{OH}$ and $\mathrm{HfP} 4$ and TaP4. For $\mathrm{O}$ adsorption, the lower $\Delta E_{O^{*}}$ are presented on the former of $3 \mathrm{~d}, 4 \mathrm{~d}$ and $5 \mathrm{~d}$ elements embedded $\mathrm{P} 4 \mathrm{C}$ than that of the later TM for the same period, respectively, which may induce the lower ORR activities referring to Norskov's theory [53]. In addition, the $\mathrm{OOH}$ group exhibits the highest adsorption free energy and the HfP4 structure has the most stability for the adsorption of $\mathrm{OOH}$. 


\subsection{Bifunctional ORR/OER catalytic activities of TMP4}

As known, the potential always deviated from the balanced potential to ensure the spontaneous reaction of ORR/OER catalytic process [54], in this work, E is defined to represent the lowest deviated potential. Typically, for ORR process, the $E_{O R R}$ is lower than the standard potential of $1.23 \mathrm{~V}$ vs. RHE at $298 \mathrm{~K}$, while the $E_{O E R}$ is higher than that. Moreover, previous study has reported that the potential gap $\left(\triangle E=E_{O E R}-E_{O R R}\right)$ is effectively suitable to present the bifunctional catalytic activity [6], and the smaller $\Delta E$ suggests the better bifunctional catalytic performance. The calculated polarization curves (describing detailly in the support information) for OER/ORR, as shown in Figure 1a, exhibit the same principle, i.e. the smaller $\Delta E$ corresponds to the higher ORR/OER catalytic efficiency. Therefore, for us, exploring novel catalysts with the lowest $\Delta E$ is still on the road.

Figure 2a displays the catalytic mechanisms of the ORR (blue) and OER (green) reaction process over TMP4, and the related $E_{O E R}, E_{O R R}$ and $\triangle E$ are calculated and shown in Figure $2 \mathrm{~b}$. For the all TMP4 catalysts, CoP4 exhibits the best OER, ORR and bifunctional catalytic activities with $E_{O E R}=1.55 \mathrm{~V}, E_{O R R}=0.86 \mathrm{~V}$ and $\Delta E=0.69 \mathrm{~V}$, respectively, which are better than that of Ni-SAC reported recently by Zhang and co-workers [52] $\left(E_{O E R}=1.62 \mathrm{~V}, E_{O R R}=0.59 \mathrm{~V}\right.$ and $\Delta E=1.03 \mathrm{~V})$. Followed by the PdP4 $\left(E_{O E R}=1.98 \mathrm{~V}, E_{O R R}=0.69 \mathrm{~V}\right.$ and $\left.\Delta E=1.29 \mathrm{~V}\right), \mathrm{RhP} 4$ $\left(E_{O E R}=1.73 \mathrm{~V}, E_{O R R}=0.07 \mathrm{~V}\right.$ and $\left.\Delta E=1.66 \mathrm{~V}\right), \mathrm{PtP} 4\left(E_{O E R}=1.79 \mathrm{~V}, E_{O R R}=0.12 \mathrm{~V}\right.$ and $\Delta E=1.67 \mathrm{~V}), \mathrm{AuP} 4\left(E_{O E R}=1.9 \mathrm{~V}, E_{O R R}=0.21 \mathrm{~V}\right.$ and $\left.\Delta E=1.69 \mathrm{~V}\right)$ and $\mathrm{NiP} 4\left(E_{O E R}=\right.$ $2.07 \mathrm{~V}, E_{O R R}=0.36 \mathrm{~V}$ and $\left.\Delta E=1.71 \mathrm{~V}\right)$. Beyond that, others show a relatively lower bifunctional catalytic activity. To further unveil the OER/ORR catalytic performance of TMP4 systems, the change of free energy of every reaction pathway was analyzed. Typically, the OER process related to four steps, including the dissociation of $\mathrm{H} 2 \mathrm{O}$ to form $* \mathrm{OH}, * \mathrm{OH}$ converts to $* \mathrm{O}$ and $\mathrm{H}+$, subsequently $* \mathrm{O}$ reacts with $\mathrm{H} 2 \mathrm{O}$ molecule to product $* \mathrm{OOH}$ and $\mathrm{H}+$, and following * $\mathrm{OOH}$ will dissociate into $\mathrm{H}+$ and $\mathrm{O} 2$, the detail reaction processes are described in Support Information. Figure 2c and Figure S2 exhibit the free energy diagrams of every OER reaction step over TMP4 systems, containing the Gibbs free energy at $\mathrm{U}=0$ and $1.23 \mathrm{~V}$. The applied external voltage will promote the energy of every reaction process shifts up and down, and the free energy of each step decreases or keeps unchanged with the direction of OER/ORR reactions sequentially 
indicating that the spontaneous catalytic reaction can be presented. Meanwhile, the $\Delta G$ was calculated to determine the potential-limited step. Results show that the free energy differences of first step for OER $\left(\Delta G_{O H^{*}}\right)$ over the TMP4 systems $(\mathrm{TM}=\mathrm{Ti}, \mathrm{V}, \mathrm{Cr}, \mathrm{Mn}, \mathrm{Zr}, \mathrm{Nb}, \mathrm{Mo}, \mathrm{Ru}, \mathrm{Hf}, \mathrm{Ta}$, $\mathrm{W}, \mathrm{Re}, \mathrm{Os}, \mathrm{Ir})$ are negative ranging from -2.15 to $-0.12 \mathrm{eV}$, demonstrating the strong bonding interactions between $\mathrm{TM}$ and $* \mathrm{OH}$ intermediate. Therefore, the third or fourth proton-coupled electron transfer steps of $* \mathrm{O}$ converting to $* \mathrm{OOH}$ and $* \mathrm{OOH}$ converting to $\mathrm{O} 2$ fragment, respectively, and the desorption of $\mathrm{O} 2$ from the TMP4 surfaces become the overpotential-limiting steps. As embedded in Figure 2 and Figure S2, the calculated overpotentials ( $\left.\eta^{\text {OER }}\right)$ of TiP4 (2.0 V), VP4 (1.76 V), CrP4 (2.01 V), MnP4 (1.57 V), ZrP4 (1.99 V), NbP4 (1.88 V), MoP4 (2.03 V), RuP4 (0.97 V), HfP4 (2.39 V), TaP4 (2.25 V), WP4 (2.23 V), ReP4 (1.75 V), OsP4 (1.72 V) and IrP4 $(0.80 \mathrm{~V})$ indicate that the high enough input energies are required for the effective OER catalytic performance. Additionally, previous study [55] has reported that the robust binding interaction and excess energy go against the elimination of related OER intermediates, impeding the active sites and poisoning the catalysts. Hence, the aforementioned TMP4 catalysts are not regarded as the available catalysts for OER. For TMP4 $(\mathrm{TM}=\mathrm{Fe}, \mathrm{Co}, \mathrm{Ni}, \mathrm{Cu}, \mathrm{Rh}, \mathrm{Pd}, \mathrm{Ag}, \mathrm{Pt}$ and $\mathrm{Au}$ ) catalysts with the positive $\Delta G_{\mathrm{OH}^{*}}$, the overpotentials are relatively lower than those of the former TMP4 systems. Specially, for the NiP4, PdP4, AgP4 and AuP4 catalysts, converting from ${ }^{*} \mathrm{OH}$ to ${ }^{*} \mathrm{O}$ for the second proton-coupled electron transfer step is unlikely due to the larger free energy difference, and which can be considered as the potential-limiting step. In contrast, the potential-limiting step of OER is the third step over FeP4, CuP4 and PtP4 surfaces and the formation of $* \mathrm{OOH}$ from $* \mathrm{O}$ is rather difficult. According to $\eta^{\mathrm{OER}}$ for FeP4 $(0.73 \mathrm{~V})$, NiP4 $(0.84$ V), CuP4 (2.93 V), PdP4 (0.75 V), AgP4 (1.44 V), PtP4 (0.56 V) and AuP4 (0.67 V), these TMP4 catalysts can also be reported inappropriate for the OER. Remarkably, the CoP4 and RhP4 catalysts exhibit outstanding OER catalytic performance, with the $\eta^{\text {OER }}$ of 0.32 and $0.50 \mathrm{~V}$, respectively, which are better than or comparable to those of Pt1/PMA (0.49 V) [55], RuO2 (0.42 V) [56], ZnN4-edge (0.63 V) [57], and the commonly used IrO2 (0.56 V) [56] catalysts. Thus, the CoP4 and RhP4 can assist as the high active catalysts for the OER. 
(a)
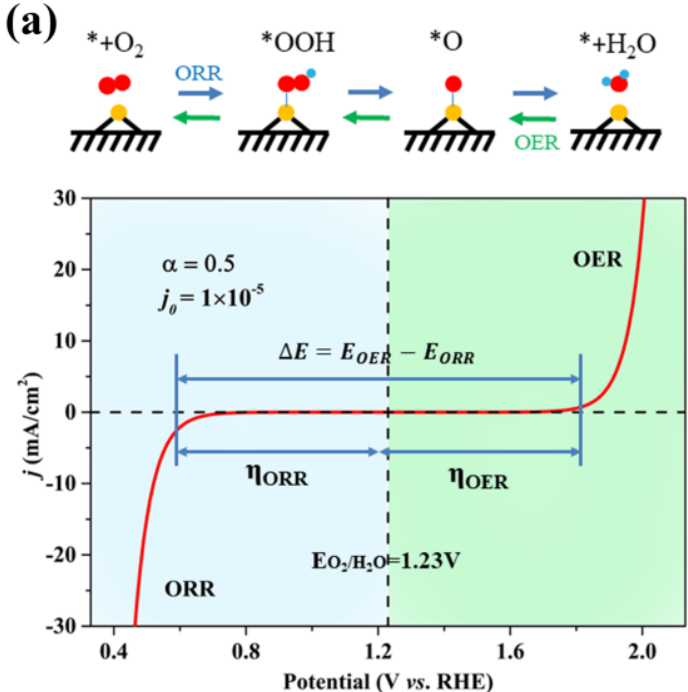

(c)

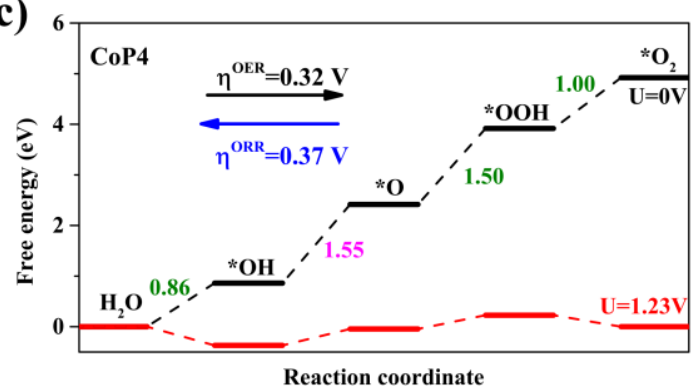

(b)
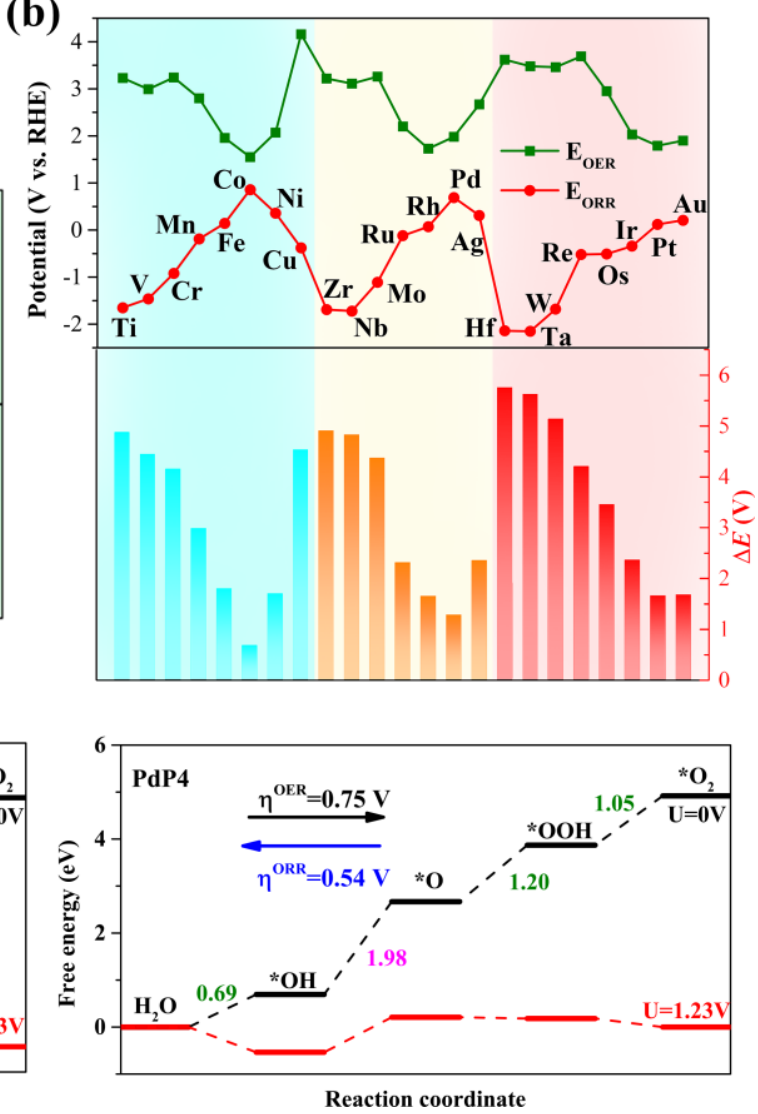

Figure 2. (a) The pathways of ORR (blue) and OER processes, the lower figure shows the theoretical polarization curves for ORR/OER catalytic performance, the detail calculated methods are shown in supporting information. (b) The related $E_{O E R}, E_{O R R}$ and $\Delta E$ for different TM-P4 systems. (c) The calculated free energy diagrams of CoP4 and PdP4 at electrode potential of 0V and $1.23 \mathrm{~V}$.

Further studies have been conducted to determine the specific ORR catalytic activities of TMP4 catalysts. It has known that the ORR is a reverse reaction of OER reaction, which also contains four proton-coupled electron transfer steps (see supporting information) on the TM active site. Therefore, Figure 2c and Figure S2 also indicate that the $\Delta G_{O H^{*}}$ of TMP4 (TM $=\mathrm{Ti}, \mathrm{V}, \mathrm{Cr}$, $\mathrm{Mn}, \mathrm{Zr}, \mathrm{Nb}, \mathrm{Mo}, \mathrm{Ru}, \mathrm{Hf}, \mathrm{Ta}, \mathrm{W}, \mathrm{Re}, \mathrm{Os}$ and Ir) for ORR process are positive and this step can be considered as the potential-limiting step with the much higher overpotentials. Meanwhile, the formation of $\mathrm{H} 2 \mathrm{O}$ will be impeded by the robust interaction between $\mathrm{OH}$ group and TMP4 catalysts for the final step of ORR. With reference to the $\eta^{\text {ORR }}$ of TiP4 $(2.88 \mathrm{~V})$, VP4 $(2.69 \mathrm{~V})$, CrP4 (2.15 V), MnP4 (1.42 V), ZrP4 (2.92 V), NbP4 (2.95 V), MoP4 (2.34 V), RuP4 (1.35 V), HfP4 (3.37 V), TaP4 (3.38 V), WP4(2.91 V), ReP4 (2.46 V), OsP4 (1.84 V) and IrP4 (1.57 V), 
high energy is required for these catalysts to proceed the ORR process, thus, these TMP4 catalysts can be abandoned for the ORR. In contrast, the systems with the negative $\Delta G_{O H^{*}}$ have the relative lower overpotentials. While, for $\mathrm{CuP} 4$ catalyst, the strong binding interaction blocks the formation of $* \mathrm{OH}$ from $* \mathrm{O}$ and brings a higher overpotentials. Furtherly, the values of $\eta^{\mathrm{ORR}}$ for CuP4 (1.61 V), FeP4 (1.09 V), NiP4 (0.87 V), RhP4 (1.16 V), PdP4 (0.54 V), AgP4 (0.92 V), PtP4 $(1.11 \mathrm{~V})$ and AuP4 $(1.02 \mathrm{~V})$ can not comparable to that of the metal Pt catalyst $(0.45 \mathrm{~V})$ [56], hence, these catalysts are also not appropriated for the ORR process. Specially, the CoP4 catalyst, with the lower ORR overpotential of $0.37 \mathrm{~V}$ and the step of * $\mathrm{OH}$ converting to $\mathrm{H} 2 \mathrm{O}$ being the potential limiting, exhibits the superior ORR catalytic performance.

\subsection{Highly reactive nature of the bifunctional catalysts}

As known, finding suitable descriptors to describe the chose conformation and exploring the correlation between these descriptors and adsorption free energies of every intermediate is an effective method to develop high performance electrocatalysts. To gain insights into the catalytic activity of TMP4 catalysts, the correlations between adsorption free energies of each intermediate containing oxygen were studied. As shown in Figure 3a, the linear relationship is existing between the adsorption free energies of $* \mathrm{OH}\left(\Delta G_{O H^{*}}\right),{ }^{*} \mathrm{O}\left(\Delta G_{O^{*}}\right)$ and ${ }^{*} \mathrm{OOH}\left(\Delta G_{O O H^{*}}\right)$ with $\Delta G_{O H^{*}}$,

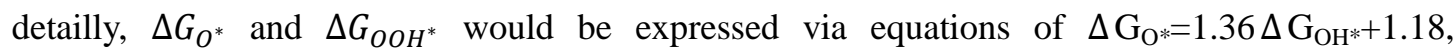
$\Delta \mathrm{G}_{\mathrm{OOH}^{*}}=0.9 \Delta \mathrm{G}_{\mathrm{OH}^{*}+3.25}$, respectively, revealing a strong correlation between $\Delta G_{O^{*}}$ and $\Delta G_{O O H^{*}}$. In addition, the volcano-curve has been extensively reported as a forceful tool for screening and developing the electrocatalysts with the high catalytic performance. As depicted in Figure 3b, showing the $\eta^{\text {OER }}$ with a descriptor of $\left(\Delta G_{O^{*}}-\Delta G_{O H^{*}}\right)$ and the $\eta^{\text {ORR }}$ with a descriptor of $\Delta G_{O H^{*}}$, respectively. Obviously, a strong correlation can be found between $\eta^{\text {OER }}$ and $\left(\Delta G_{O^{*}}-\Delta G_{O H^{*}}\right)$, implying that the second proton-coupled electron transfer step is the key step for the OER over most TMP4 catalysts. Meanwhile, the strong correlation also exists between $\eta^{\mathrm{ORR}}$ and $\Delta G_{O H^{*}}$, demonstrating that the desorption of $* \mathrm{OH}$ plays a key role in the ORR catalytic process. For the both volcanos, $\mathrm{CoP} 4$ catalyst occupies the top owing to the moderate adsorption interaction between TMP4 and OER/ORR intermediates, thus performing the highest activity for the OER/ORR process with the minimum $\eta^{\text {OER }}(0.32 \mathrm{~V})$ and $\eta^{\text {ORR }}(0.37 \mathrm{~V})$. Therefore, the CoP4 can be regarded as the promising bifunctional OER/ORR electrocatalyst and better than $\mathrm{IrO} 2$ and $\mathrm{Pt}$ 
catalysts. The optimized stable structures of reaction intermediates adsorbed on CoP4 are displayed in Figure 3d.
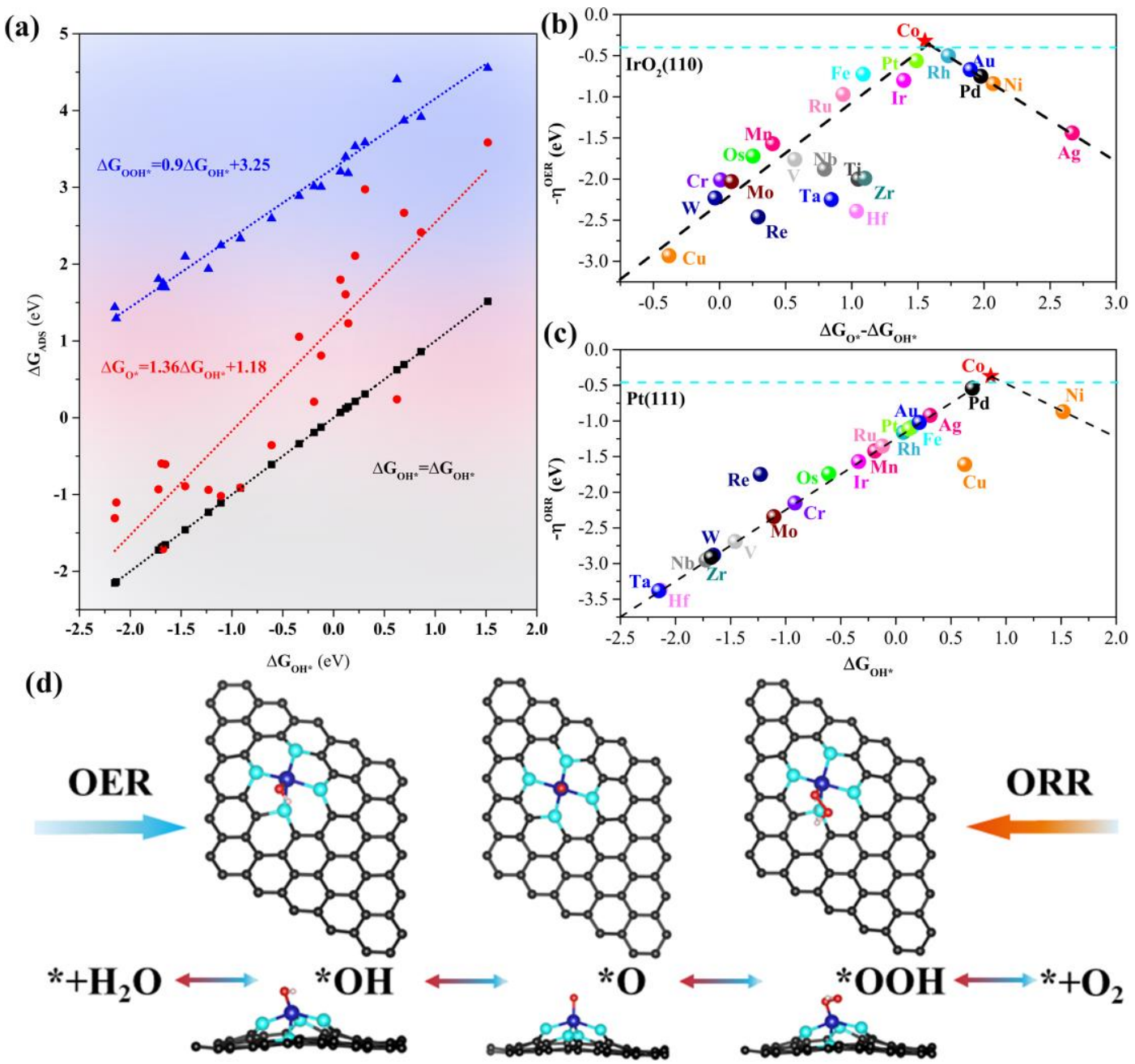

\section{ORR}

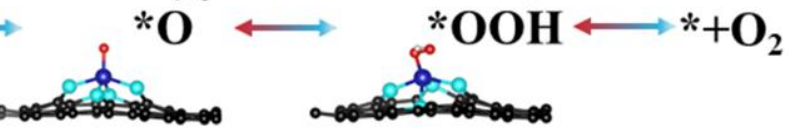

Figure 3. (a) Calculated $\Delta G_{O H^{*}}, \Delta G_{O O H^{*}}$ and $\Delta G_{O^{*}}$ compared with $\Delta G_{O H^{*}}$ for each TMP4 catalysts. Volcano curves for (b) $-\eta^{\text {OER }}$ vs. $\Delta G_{O^{*}}-\Delta G_{O H^{*}}$ and (c) $-\eta^{\text {ORR }}$ vs. $\Delta G_{O H^{*}}$ for different

TMP4 catalysts, blue lines represent the overpotentials of $\mathrm{IrO}$ (110) and $\mathrm{Pt}(111)$ catalysts,

respectively. (d) displays the stable configurations of $* \mathrm{OH},{ }^{*} \mathrm{OOH}$ and $* \mathrm{O}$ adsorbed on CoP4.

Besides that, the electronic properties of all TMP4 catalysts were calculated to evaluate the reactivity and mechanism of bifunctional OER/ORR process. Figure 4a and Figure S3 exhibit the d-orbital DOS of the embedded TM atoms for different TMP4 systems. Previous reports $[58,59]$ have announced that the d-band center $\left(E_{d}\right.$, obtaining from $d$ orbital of $T M$ atom) can be extensively used to furtherly expose the catalytic properties, therefore, which is labeled in the plots of d-orbital DOS. Results suggest that, in the same period, the position of $E_{d}$ will move 
toward to a more negative value relative to Fermi level with the increased d electron numbers of TM atoms, accordingly, causing the changed adsorption strengthen of every intermediate over TMP4 catalysts. Generally, the adsorption is weaker with a more negative $\mathrm{E}_{\mathrm{d}}$ of TM atom, which can be explained by the hybridization between TM-d orbital and the electron states of adsorption species. As displayed in Figure 4b, the hybridization of orbitals promotes the production of antibonding and bonding states. And the antibonding states are highly occupied when the $E_{d}$ is located at a more negative value, which weakens the adsorption. Moreover, the high concentrations of $\mathrm{d}$ free electrons near Fermi level will devote rich electrons to reaction intermediates, resulting in the strong adsorptions and challenges for produce releasing (such as TiP4 system). In constant, the low d-electrons concentrations of catalysts could not maintain the effective adsorption of reaction intermediates (AgP4). Hence, for OER/ORR process, a suitable $\mathrm{E}_{\mathrm{d}}$ position (a modest adsorption strengthen of intermediates) plays a key role for screening catalysts. In addition, Figure $4 \mathrm{c}$ and (d) show the correlations between bifunctional catalytic activity $(\Delta E)$ and $\mathrm{E}_{\mathrm{d}}$, work function ( $\Phi$, which can imply the ability of charge transfer for configuration), indicating that the closer to $-1.53 \mathrm{~V}$ the $\mathrm{E}_{\mathrm{d}}$ is or the larger the work function is, the better the bifunctional catalytic activity is. In summary, the CoP4 with the suitable adsorption strengthen exhibits the best bifunctional catalytic performance due to the optimized electronic structure, which is a result of the synergistic effect with a modest $E_{d}$ and a large work function for Co atom. The PDOS and charge density difference [60] of intermediates adsorption (Figure S4) on CoP4 indicate that the adsorption attributes to the charges transfer, originating from the hybridization between Co-d and p orbitals of oxygen - containing groups. 
(a)

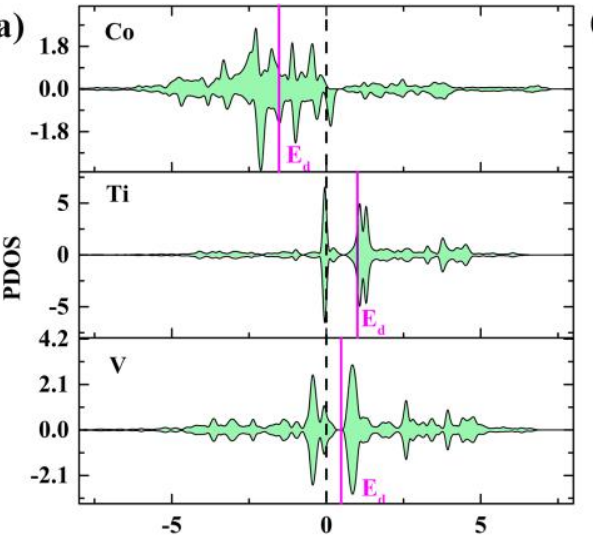

(b)

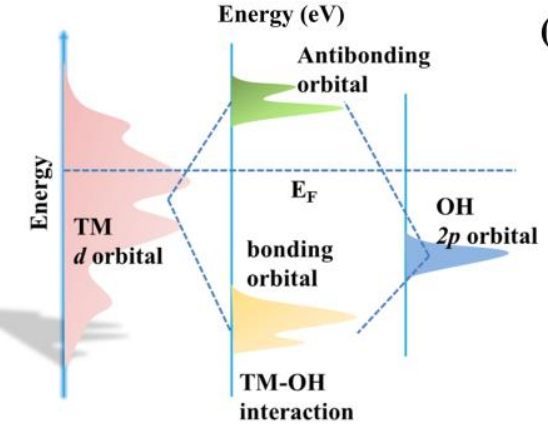

(c)
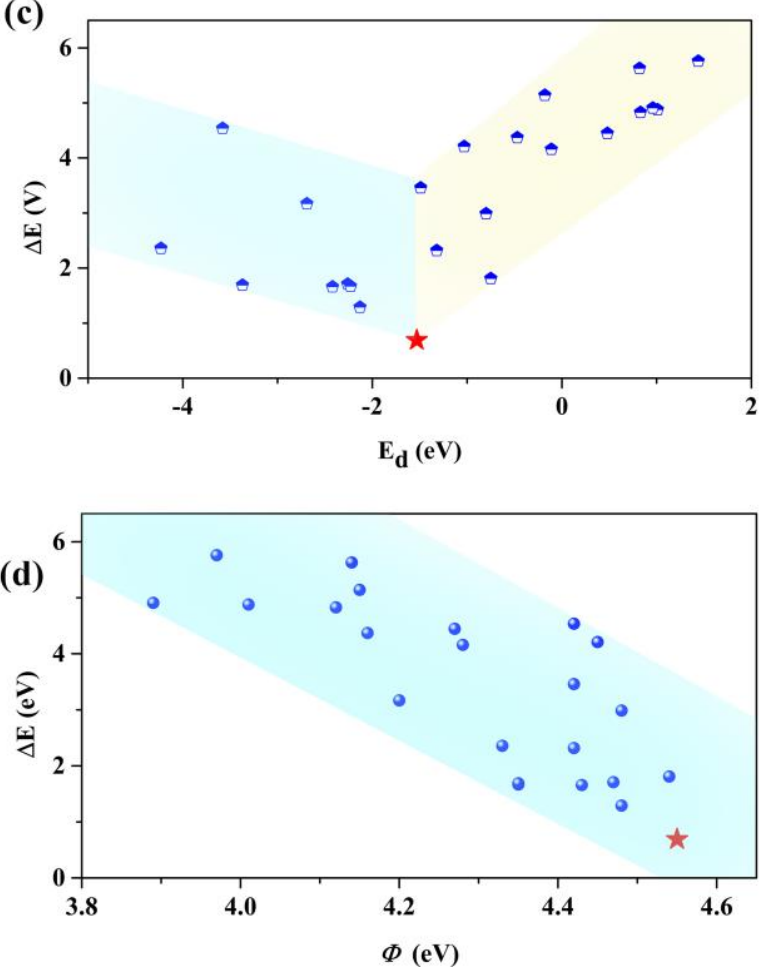

Figure 4. (a) Partial density of states (PDOS) of d orbitals for Co, Ti and V embedded P4C systems, dotted and red lines represent the Fermi level and the d-band center $\left(E_{d}\right)$ of TM atoms, respectively. (b) The schematic diagram demonstrating how TM atom interact with OH onTMP4. (c) and (d) display the correlation between $\mathrm{E}_{\mathrm{d}}$, work function and change in bifunctional potential $\Delta E$ for the TMP4 systems.

In the last, the thermodynamic stability of $\mathrm{CoP} 4$ catalyst is then evaluated by AIMD simulations in the NVT ensemble $(300 \mathrm{~K})$. As shown in Figure $5 \mathrm{a}$ and $\mathrm{b}$, the energy and temperature oscillate near the initial values, and the structure of $\mathrm{CoP} 4$ is preserved well (Figure 5c) after $300 \mathrm{~K}$ dynamic simulation for $10 \mathrm{ps}$. Therefore, it can be concluded that the CoP4 serves as an efficient bifunctional OER/ORR catalyst with high stability. 


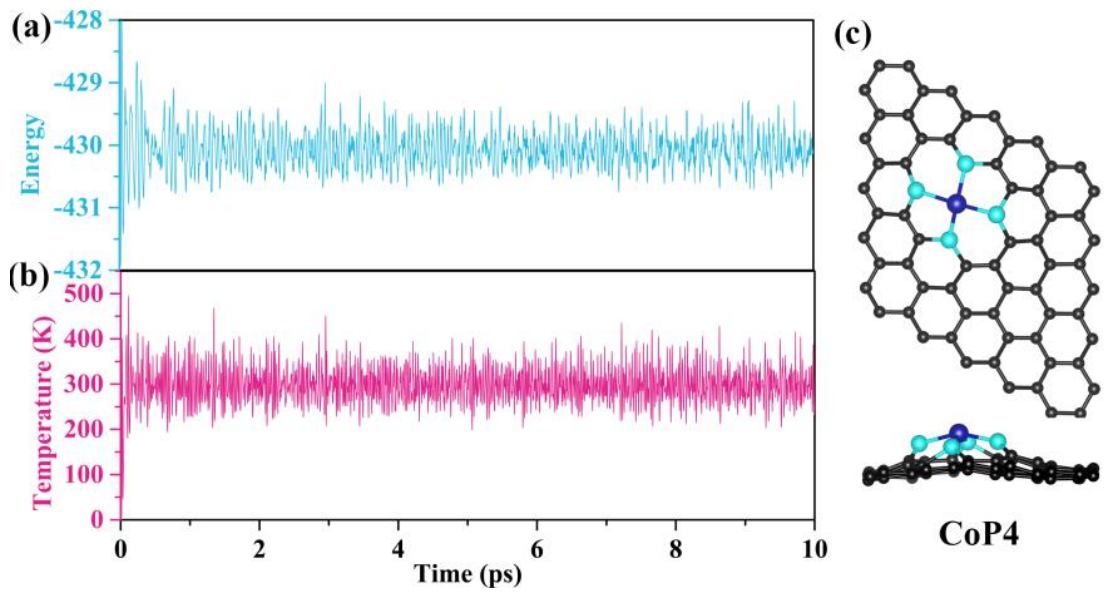

Figure 5. (a)Total energy and (b) temperature evolution versus the time for $\mathrm{CoP} 4$ catalyst, and the simulation time is $10 \mathrm{ps}$ at $300 \mathrm{~K}$. (c) exhibits the geometric structure of CoP4 after 10 ps AIMD.

\section{Conclusions}

In this work, the application potential of transition metal and P co-doped graphene-based SACs (TMP4) as the bifunctional ORR/OER electrocatalysts has been comprehensively explored by using first-principle calculations. Results demonstrate that the negative binding energies between TM and P4C can guarantee the stability of the structures of TMP4. Then, the bifunctional ORR/OER activity of TMP4 were evaluated, and the CoP4 structure, whose bifunctional catalytic activity are even better than that of $\mathrm{Pt}$ and $\mathrm{IrO}_{2}$ catalysts, exhibits the most promising bifunctional ORR and OER catalytic performances with the low overpotentials of 0.37 and $0.32 \mathrm{eV}$ (the smallest $\Delta E$ ), respectively. Based on the scaling relation between $\mathrm{OH}^{*}, \mathrm{O}^{*}$ and $\mathrm{OOH}^{*}$, we established the volcano plots to suggest the activity tendency of ORR and OER processes on TMP4, showing that CoP4 catalyst occupies the top owing to the moderate adsorption interaction between TMP4 and OER/ORR intermediates. In addition, the PDOS, d-band center $\left(\mathrm{E}_{\mathrm{d}}\right)$ and work function were calculated to clarify the origin of highly reactive nature of TMP4. Furthermore, the AIMD simulation was conducted to evaluate and guarantee the thermodynamic stability of $\mathrm{CoP} 4$ catalyst. Therefore, our study theoretically accentuates that $\mathrm{CoP} 4$ is a promising electrocatalyst for ORR and OER with high activity and stability.

\section{SUPPORTING INFORMATION}

Supporting figures and tables. And the detail description of the calculation methods, including the 
reaction pathways of ORR and OER, the free energy change of each step, the overpotential for

ORR and OER, and the measurement of polarization curves.

\section{ACKNOWLEDGMENTS}

This work was supported by the National Natural Science Foundation of China (51602196, 52072240), the Shanghai Automotive Industry Corporation (1714), and the Materials Genome Initiative Center at Shanghai Jiao Tong University. All simulations were performed at the Shanghai Jiao Tong University High Performance Computing Center.

\section{References}

[1] H. Li, Z. Wang, L. Chen, X. Huang, Adv. Mater. 2009, 21, 4593-4607.

[2] M. K. Debe, Nature 2012, 486, 43-51.

[3] Z. Chang, X. Zhang, Introduction to Metal-Air Batteries: Theory and Basic Principles, Vol. 1, Wiley-VCH, Weinheim, Germany 2018.

[4] L. Li, A. Manthiram, Adv. Energy Mater. 2016, 6, 1502054

[5] J. Lee, S. Tai Kim, R. Cao, N. Choi, M. Liu, K. T. Lee, J. Cho, Adv. Energy Mater. 2011, 1, 34.

[6] Z. Huang, J. Wang, Y. Peng, C. Jung, A. Fisher, X. Wang, Adv. Energy Mater. 2017, 7, 1700544.

[7] L. Li, X. Zhao, A. Manthiram, Electrochem. Commun. 2012, 14, 78.

[8] A. Kraytsberg, Y. Ein-Eli, Nano Energy 2013, 2, 468.

[9] Y. Jiao, Y. Zheng, M. Jaroniec, S. Z. Qiao, Chem. Soc. Rev. 2015, 44, 2060.

[10] H. G. Sanchez Casalongue, M. L. Ng, S. Kaya, D. Friebel, H. Ogasawara, A. Nilsson, Angew. Chem., Int. Ed. 2014, 53, 7169.

[11] V. Tripkovic, Phys. Chem. Chem. Phys. 2017, 19, 29381.

[12] Z.W. Seh, J. Kibsgaard, C.F. Dickens, I. Chorkendorff, J.K. Nørskov, T.F. Jaramillo, Seh, Z. W.; Kibsgaard, J.; Dickens, C. F.; Chorkendorff, I.; Nørskov, J. K.; Jaramillo, T. F. Science 2017, 146, eaad4998.

[13] Y. Peng, B. Lu and S. Chen, Adv. Mater., 2018, 30, 1801995

[14] Z. Wang, J. Zhao, Q. Cai and F. Li, J. Mater. Chem. A, 2017, 5, 9842-9851

[15] Q. Peng, J. Zhou, J. Chen, T. Zhang and Z. Sun, J. Mater. Chem. A, 2019, 7, 26062-26070

[16] Qu, K.; Zheng, Y.; Dai, S.; Qiao, S. Z. Nano Energy 2016, 19, 373-381.

[17] Qian, L.; Lu, Z.; Xu, T.; Wu, X.; Tian, Y.; Li, Y.; Huo, Z.; Sun, X.; Duan, X. Adv. Energy Mater. 2015, 5, 1500245.

[18] Q.M. Deng, J. Zhao, T. Wu, G. Chen, H.A. Hansen, T.Vegge, Journal of Catalysis, 2019, 370, 378-384.

[19] W. Ju, A. Bagger, G.-P. Hao, A. S. Varela, I. Sinev, V. Bon, B. R. Cuenya, S. Kaskel, J. Rossmeisl and P. Strasser, Nat. Commun., 2017, 8, 944.

[20] H. J. Qiu, Y. Ito, W. Cong, Y. Tan, P. Liu, A. Hirata, T. Fujita, Z. Tang and M. Chen, Angew. Chem., Int. Ed., 2015, 54, 14031-14035.

[21] L. Zhang, Y. Jia, G. Gao, X. Yan, N. Chen, J. Chen, M. T. Soo, B. Wood, D. Yang, A. Du and X. Yao, Chem, 2018, 4, 285-297 
[22] H. Yan, H. Cheng, H. Yi, Y. Lin, T. Yao, C. Wang, J. Li, S. Wei and J. Lu, J. Am. Chem. Soc., 2015, 137, 10484-10487

[23] A. Zitolo, V. Goellner, V. Armel, M.-T. Sougrati, T. Mineva, L. Stievano, E. Fonda, F. Jaouen, Nat. Mater. 14 (2015) 937.

[24] X. Fu, N. Li, B. Ren, G. Jiang, Y. Liu, F.M. Hassan, D. Su, J. Zhu, L. Yang, Z. Bai, Z.P. Cano, A. Yu, Z. Chen, Adv. Energy Mater. 9(11) (2019) 1803737.

[25] X. Zhang, Z. Yang, Z. Lu, W. Wang, Carbon 130 (2018) 112-119.

[26] H. Yan, Y. Lin, H. Wu, W. Zhang, Z. Sun, H. Cheng, W. Liu, C. Wang, J. Li, X. Huang, T. Yao, J. Yang, S. Wei and J. Lu, Nat. Commun., 2017, 8, 1070

[27] H. Xu, D. Cheng, D. Cao and X. C. Zeng, Nat. Catal., 2018, 1, 339-348.

[28] X. Guo, S. Lin, J. Gu, S. Zhang, Z. Chen and S. Huang, ACS Catal., 2019, 9, 11042-11054.

[29] S. Lin, H. Xu, Y. Wang, X. C. Zeng and Z. Chen, J. Mater. Chem. A, 2020, 8, 5663-5670

[30] Zhang X, Yang Z, Lu Z, Wang W., Carbon, 2018,130, 112-119.

[31] Y. Li, X. Liu, L. Zheng, J. Shang, X. Wan, R. Hu, X. Guo, S. Hong, J. Shui, J. Mater. Chem. A 2019, 7, 26147.

[32] Z. Lu, G. Xu, C. He, T. Wang, L. Yang, Z. Yang, D. Ma, Carbon 84 (2015) 500-508.

[33] J. Li, S. Chen, N. Yang, M. Deng, S. Ibraheem, J. Deng, J. Li, L. Li, Z. Wei, Angew. Chem., Int. Ed. 2019, 58, 7035

[34] F. Li, G.F. Han, H.J. Noh, S.J. Kim, Y. Lu, H.Y. Jeong, Z. Fu, J.B. Baek, Energy Environ. Sci., 2018, 11, 2263-2269.

[35] Z. Liang, M. Luo, M. Chen, X. Qi, J. Liu, C. Liu, S.G. Peera, T.X. Liang, J. Mater. Chem. A, 2020, 8, 20453-20462.

[36] G. Zhang, Y. Jia, C. Zhang, X. Xiong, K. Sun, R. Chen, W. Chen, Y. Kuang, L. Zheng, H. Tang, W. Liu, J. Liu, X. Sun, F. Lin and H. Dai, Energy Environ. Sci., 2019, 12, 1317-1325

[37] Y. Lin, P. Liu, E. Velasco, G. Yao, Z. Tian, L. Zhang and L. Chen, Adv. Mater., 2019, 31, 1808193.

[38] Y. Mun, S. Lee, K. Kim, S. Kim, S. Lee, J. W. Han and J. Lee, J. Am. Chem. Soc., 2019, 141, 6254-6262.

[39] T. Sun, Y. Li, T. Cui, L. Xu, Y.G. Wang, W. Chen, P. Zhang, T. Zheng, X. Fu, S. Zhang, Z. Zhang, D. Wang, Y. Li, Nano Lett. 2020, 20, 6206-6214.

[40] R. Li, Z.D. Wei, X.L. Gou, W. Xu, RSC Adv. 2013, 3, 9978.

[41] D.S. Yang, D. Bhattacharjya, S. Inamdar, J. Park, J.S. Yu, J. Am. Chem. Soc. 2012, 134, 16127.

[42] Z.W. Liu, F. Peng, H.J. Wang, H. Yu, W.X. Zheng, J. Yang, Angew. Chem. Int. Ed. 2011, 50, 3257

[43] J. Wu, Z.R. Yang, Z.J. Wang, Q.J. Sun, R.Z. Yang, Electrochem. Commun. 2014, 42, 46-49.

[44] Z. Yang, J. Wu, X. Zheng, Z. Wang, R. Yang, J. Power Sources 2015, 277, 161-168.

[45] F. Razmjooei, K. P. Singh, E.J. Bae, J.S. Yu, J. Mater. Chem. A, 2015, 3, 11031-11039.

[46] G. Kresse, J. Furthmüller, Phys. Rev. B 1996, 54, 11169

[47] J. P. Perdew, Y. Wang, Phys. Rev. B 1992, 45, 13244.

[48] J. P. Perdew, K. Burke, M. Ernzerhof, Phys. Rev. Lett. 1996, 77, 3865

[49] K. Lee, É. D. Murray, L. Kong, B. I. Lundqvist, D. C. Langreth, Phys. Rev. B 2010, 82, 081101.

[50] K. Mathew, R. Sundararaman, K. Letchworth-Weaver, T. Arias, R. G. Hennig, J. Chem. Phys. 
2014, 140, 084106

[51] H. Niu, X.H. Wan, X. Wang, C. Shao, J. Robertson, Z.F. Zhang, Y.Z. Guo, ACS Sustainable Chem. Eng. 2021, 9, 3590-3599.

[52] H. Niu, Z.F. Zhang, X. Wang, X. Wan, C. Shao, Y.Z. Guo, Adv. Func. Mater. 2021, 31, 2008533.

[53] J. K. Nørskov, J. Rossmeisl, A. Logadottir, L. L, J. Phys. Chem. B 2004, 108, 17886

[54] B. Wei, Z.H. Fu, D. Legut, T,C, Germann, S.Y. Du, H.J. Zhang, J.S. Francisco, R.F. Zhang, Adv. Mater. 2021, 2102595.

[55] S.H. Talib, Z. Lu, X. Yu, K. Ahmad, B. Bashir, Z. Yang, J. Li, ACS Catalysis 2021, 11, 8929-8941

[56] I. C. Man, H.Y. Su, F. Calle-Vallejo, H. A. Hansen, J. I. Martínez, N. G. Inoglu, J. Kitchin, T. F. Jaramillo, J.K. Nørskov, J. Rossmeisl, ChemCatChem 2011, 3, 1159-1165.

[57] Y. Li, R. Hu, X. Wan, J.X. Shang, F.H. Wang, J. Shui, Adv. Theory Simul. 2020, 3, 2000054.

[58] C. Ling, L. Shi, Y. Ouyang, X.C. Zeng, J. Wang, Nano Lett. 2017, 17, 5133-5139.

[59] Z. Fu, C. Ling, J. Wang, J. Mater. Chem. A 2020, 8, 7801-7807.

[60] Z. Xu, X. Zhang, J. Qin, R. Liu, J. Energy Chem. 2021, 55, 437-443. 